\title{
Identification and data-driven reduced-order modeling for linear conservative port- and self-adjoint Hamiltonian systems
}

\author{
Paolo Rapisarda and Arjan van der Schaft
}

\begin{abstract}
Given a sufficiently numerous set of vectorexponential trajectories of a conservative port-Hamiltonian system and the supply rate, we compute a corresponding set of state trajectories by factorizing a constant Pick-like matrix. State equations are then obtained by solving a system of linear equations involving the system trajectories and the computed state ones. If a factorization of only a principal submatrix of the Pick matrix is performed, our procedure yields a lower-order conservative port-Hamiltonian model obtained by projection of the full-order one. We also describe a similar approach to identification and model-order reduction for self-adjoint Hamiltonian systems.
\end{abstract}

Index Terms-conservative port-Hamiltonian systems; selfadjoint Hamiltonian systems; behaviors; quadratic differential forms; rank-revealing factorization.

\section{INTRODUCTION}

Denote by $\exp (\lambda)$ the exponential function whose value at $t \in \mathbb{R}$ is $e^{\lambda t}$; given $N$ (input, output) vector-exponential trajectories

$$
\left\{\left[\begin{array}{l}
u_{i} \\
y_{i}
\end{array}\right] \exp \left(\lambda_{i}\right) \mid u_{i}, y_{i} \in \mathfrak{C}^{\infty}\left(\mathbb{R}, \mathbb{R}^{m}\right)\right\}_{i=1, \ldots, N}
$$

originating from a conservative linear port-Hamiltonian system (see for the precise definition later on), we want to identify a state-space model

$$
\begin{aligned}
\frac{d}{d t} x & =A x+B u \\
y & =C x+D u,
\end{aligned}
$$

compatible with the data. In this paper we show that in order to do this, we can first compute vectors $x_{i} \in \mathbb{C}^{n}$ such that for $i=1, \ldots, N, x_{i} \exp \left(\lambda_{i}\right), u_{i} \exp \left(\lambda_{i}\right), y_{i} \exp \left(\lambda_{i}\right)$ satisfy (2). These are obtained by factorizing in a rank-revealing way the Pick matrix, i.e. the Hermitian matrix whose $(i, j)$-th entry is

$$
P_{i, j}:=\frac{\left[\begin{array}{ll}
u_{i}^{*} & y_{i}^{*}
\end{array}\right]\left[\begin{array}{cc}
0_{m \times m} & I_{m} \\
I_{m} & 0_{m \times m}
\end{array}\right]\left[\begin{array}{l}
u_{i} \\
y_{i}
\end{array}\right]}{\lambda_{i}^{*}+\lambda_{j}},
$$

i.e. $P=\left[\begin{array}{lll}x_{1} & \ldots & x_{N}\end{array}\right]^{*} Q\left[\begin{array}{lll}x_{1} & \ldots & x_{N}\end{array}\right]$. To determine the matrices $A, B, C, D$, we then solve the system of $N+m$ equations

$$
\left[\begin{array}{ccc}
x_{1} \lambda_{1} & \ldots & x_{N} \lambda_{N} \\
y_{1} & \ldots & y_{N}
\end{array}\right]=\left[\begin{array}{cc}
A & B \\
C & D
\end{array}\right]\left[\begin{array}{lll}
x_{1} & \ldots & x_{N} \\
u_{1} & \ldots & u_{N}
\end{array}\right] .
$$

P. Rapisarda is with the CSPC group, School of Electronics and Computer Science, University of Southampton, Great Britain, e-mail: pr3eecs.soton.ac.uk, Tel: +(44)2380593367, Fax: +(44)2380594498; Arjan van der Schaft is with the Johann Bernoulli Institute for Mathematics and Computer Science, University of Groningen, PO Box 407, 9700 AK, the Netherlands, e-mail: a.j.van.der.schafterug.nl
Among the solutions $A, B, C, D$ of (4) there are matrices corresponding to a state-space realisation of a conservative port-Hamiltonian system associated with the data $u_{i}, y_{i}$, $i=1, \ldots, N$. Consequently if $N<n$, our procedure yields a reduced-order conservative port-Hamiltonian model of McMillan degree $N$, whose matrices $A^{\prime}, B^{\prime}, C^{\prime}, D^{\prime}$ are obtained by projecting the state matrices $A, B, C, D$ of a full-order state representation.

Compared with standard identification algorithms (e.g. the family of subspace identification algorithms, see [9]) and with recent work on structure-preserving model-order reduction based on interpolation techniques (see [1], [11]), the approach presented in this paper is novel in two aspects. From the identification point of view, we compute state trajectories exploiting the existence of a bilinear form on the external (i.e. input-output) variables of the system; such bilinear form is structurally connected to a bilinear form on the internal (i.e. state) variables. Contrast this approach with standard subspace identification algorithms, whose foundation is essentially the time-invariant nature of the system.

From the model reduction point of view, our starting point is not a given (state- or otherwise) representation of the system, but rather data gathered in controlled experiments on the unknown system.

In this paper we will use the behavioral framework, see [10], [13]; the calculus of quadratic differential forms (QDFs), see [18]; and the framework of port-Hamiltonian systems and Dirac structures, see [15], [14], [11]. A summary of the relevant notions is given in Section II and Appendix VIII. In Section III we investigate some properties of the Pick matrix (3); in Sections IV and V we illustrate our approach to the identification and model-order reduction of conservative port-Hamiltonian systems. Finally, we extend the framework to self-adjoint Hamiltonian systems in Section VI.

\section{Notation}

The space of real vectors with $n$ components is denoted by $\mathbb{R}^{n}$, and the space of $n \times m$ real matrices by $\mathbb{R}^{m \times n}$. If $A_{1}, \ldots, A_{n}$ are matrices with the same number of columns, we denote by $\operatorname{col}\left(A_{1}, \ldots, A_{n}\right)$ the matrix obtained by stacking $A_{i}, i=1 \ldots, n$ on top of each other. The ring of polynomials with real coefficients in the indeterminate $\xi$ is denoted by $\mathbb{R}[\xi]$; the ring of two-variable polynomials with real coefficients in the indeterminates $\zeta$ and $\eta$ is denoted by $\mathbb{R}[\zeta, \eta] . \mathbb{R}^{n \times m}[\xi]$ is the space of $n \times m$ polynomial matrices in $\xi$, and the space of $n \times m$ polynomial matrices in $\zeta$ and $\eta$ is denoted by $\mathbb{R}^{n \times m}[\zeta, \eta]$. The set of infinitely-differentiable functions from $\mathbb{R}$ to $\mathbb{R}^{w}$ is denoted by $\mathfrak{C}^{\infty}\left(\mathbb{R}, \mathbb{R}^{w}\right)$. 


\section{BACKGROUND}

\section{A. Behaviors}

We consider systems represented in image representation, i.e. there exists $M \in \mathbb{R}^{2 m \times m}[\xi]$ such that $w=\left[\begin{array}{l}u \\ y\end{array}\right] \in$ $\mathfrak{C}^{\infty}\left(\mathbb{R}, \mathbb{R}^{m+m}\right)$ belongs to the system if and only if there exists $\ell \in \mathfrak{C}^{\infty}\left(\mathbb{R}, \mathbb{R}^{m}\right)$ such that

$$
w=M\left(\frac{d}{d t}\right) \ell
$$

We assume that the polynomial matrix $M(\lambda) \in \mathbb{C}^{2 m \times m}$ has full column rank $m$ for all $\lambda \in \mathbb{C}$. We also assume that $u$ is an input-, and $y$ an output variable; then we can write

$$
M=\left[\begin{array}{l}
D \\
N
\end{array}\right]
$$

with $D \in \mathbb{R}^{m \times m}[\xi]$ nonsingular.

For any $X \in \mathbb{R}^{n \times m}[\xi]$ the differential operator $X\left(\frac{d}{d t}\right)$ : $\mathfrak{C}^{\infty}\left(\mathbb{R}, \mathbb{R}^{m}\right) \rightarrow \mathfrak{C}^{\infty}\left(\mathbb{R}, \mathbb{R}^{n}\right)$ given by $x=X\left(\frac{d}{d t}\right) \ell$ is a state map for the set of trajectories described by (5) if for all $\left[\begin{array}{l}u_{i} \\ y_{i}\end{array}\right]$ with associated latent variable trajectories $\ell_{i}$ satisfying (5), $i=1,2$ and corresponding $x_{i}=X\left(\frac{d}{d t}\right) \ell_{i}, i=1,2$, the state property holds [13], [16]. In this case $x:=X\left(\frac{d}{d t}\right) \ell$ is called a state variable. If $n$ is minimal among all the state dimensions, then $X\left(\frac{d}{d t}\right)$ is a minimal state map, and the number of its rows is the McMillan degree of the system.

\section{B. Quadratic differential forms}

Let $\Phi \in \mathbb{R}^{\mathrm{w} \times \mathrm{w}}[\zeta, \eta]$ be a two-variable polynomial matrix, i.e. $\Phi(\zeta, \eta)=\sum_{k, \ell=0} \Phi_{k, \ell} \zeta^{k} \eta^{\ell}$, where $\Phi_{k, \ell} \in \mathbb{R}^{\mathrm{w} \times \mathrm{w}}$, $k, \ell \in \mathbb{N}$, and the sum extends over a finite set of indices. The quadratic differential form (QDF) $Q_{\Phi}: \mathfrak{C}^{\infty}\left(\mathbb{R}, \mathbb{R}^{\mathrm{w}}\right) \rightarrow$ associated with $\Phi(\zeta, \eta)$ is defined by

$$
w \mapsto Q_{\Phi}(w)=\sum_{k, \ell}\left(\frac{d^{k}}{d t^{k}} w\right)^{\top} \Phi_{k, \ell}\left(\frac{d^{\ell}}{d t^{\ell}} w\right) .
$$

In the following without loss of generality we assume that $\Phi(\zeta, \eta)=\Phi(\eta, \zeta)^{\top}$, i.e. that $\Phi(\zeta, \eta)$ is symmetric. The derivative $\frac{d}{d t} Q_{\Phi}=: Q_{\Phi}$ of a QDF $Q_{\Phi}$ is also a QDF, and the associated two-variable polynomial matrix is $(\zeta+\eta) \Phi(\zeta, \eta)$.

\section{Conservative port-Hamiltonian systems}

Consider the non-degenerate bilinear form $Q_{e}$ on $\mathbb{R}^{2 m}$

$$
Q_{e}=\left[\begin{array}{ll}
0_{m} & I_{m} \\
I_{m} & 0_{m}
\end{array}\right] \text {. }
$$

induced, see Appendix VIII, by the Euclidean inner product on $\mathbb{R}^{m}$. This gives rise to a non-degenerate bilinear form on the set $\mathcal{C}\left(\mathbb{R}, \mathbb{R}^{2 m}\right)$ of piecewise right-continuous functions $w: \mathbb{R} \rightarrow \mathbb{R}^{2 m}$ with compact support:

$$
\left\langle\left\langle w_{1}, w_{2}\right\rangle\right\rangle:=\int_{-\infty}^{\infty} w_{1}^{T}(t) Q_{e} w_{2}(t) d t .
$$

Recall from Appendix VIII the definition of a Dirac structure; the definition of a linear conservative linear port-Hamiltonian system is the following (see [16]).
Definition 1: Consider the image representation (5); $\operatorname{im} M\left(\frac{d}{d t}\right)$ is a conservative port-Hamiltonian system if it is a Dirac structure with respect to the bilinear form $\langle\langle\cdot, \cdot\rangle\rangle$ on the linear space $\mathcal{C}\left(\mathbb{R}, \mathbb{R}^{2 m}\right)$.

Thus if im $M\left(\frac{d}{d t}\right)$ is conservative port-Hamiltonian then

$$
\begin{aligned}
& {\left[\begin{array}{ll}
u(t)^{\top} & y(t)^{\top}
\end{array}\right] Q_{e}\left[\begin{array}{l}
u(t) \\
y(t)
\end{array}\right] d t} \\
& =\int_{-\infty}^{\infty}\left(M\left(\frac{d}{d t}\right) \ell_{1}(t)\right)^{\top} Q_{e} M\left(\frac{d}{d t}\right) \ell_{2}(t)=0
\end{aligned}
$$

for all $\ell_{1}, \ell_{2}$ with compact support, and furthermore the system is maximal with regard to this property. The following theorem is a reformulation of the results presented in [16], more suitable to the purposes of this paper.

Theorem 1: Let $X \in \mathbb{R}^{n \times m}[\xi]$ be a minimal state map for the system represented by (5). Assume that im $M\left(\frac{d}{d t}\right)$ is conservative port-Hamiltonian. Then there exists an invertible $Q=Q^{\top} \in \mathbb{R}^{n \times n}$ such that $M(\zeta)^{\top} J M(\eta)=$ $(\zeta+\eta) X(\zeta)^{\top} Q X(\eta)$.

Theorem 1 implies that for every $\operatorname{col}\left(u_{i}, y_{i}\right)=M\left(\frac{d}{d t}\right) \ell_{i}$ with state trajectories $x_{i}:=X\left(\frac{d}{d t}\right) \ell_{i}, i=1,2$

$$
\frac{d}{d t} x_{1}^{T} Q x_{2}=\operatorname{col}\left(u_{1}, y_{1}\right)^{\top} Q_{e} \operatorname{col}\left(u_{2}, y_{2}\right) .
$$

The quadratic form $\frac{1}{2} x^{T} Q x$ defines the energy of the system, $\frac{1}{2} \operatorname{col}(u, y)^{\top} Q_{e} \operatorname{col}(u, y)$ defines the standard passivity supply rate, while (10) amounts to the energy balance

$$
\frac{d}{d t} \frac{1}{2} x^{\top} Q x=\frac{1}{2}\left(u^{\top} y+y^{\top} u\right)=u^{T} y,
$$

A minimal input-state-output representation of a conservative port-Hamiltonian system takes the form [15], [14], [6], [16]

$$
\begin{aligned}
\frac{d}{d t} x & =J Q x+B u \\
y & =B^{\top} Q x+M u,
\end{aligned}
$$

where $J=-J^{\top} \in \mathbb{R}^{n \times n}$ and $M=-M^{\top} \in \mathbb{R}^{m \times m}$.

\section{THE PICK MATRIX AND ITS PROPERTIES}

We are given $N$ pairs $\left(\lambda_{i}, v_{i}\right), i=1, \ldots, N$, where $v_{i} \in$ $\mathbb{C}^{m}, \lambda_{i} \in \mathbb{C}$ with the property that $\lambda_{i}$ does not lie on the imaginary axis, $\lambda_{i} \neq \lambda_{j}$ for $i \neq j$, and $\lambda_{j}=\lambda_{i}^{*} \Rightarrow v_{j}=\overline{v_{i}}$. Given the observable image representation (5) partitioned as in (6), our data-set is defined by

$$
\left\{\left[\begin{array}{l}
u_{i} \\
y_{i}
\end{array}\right] \exp \left(\lambda_{i}\right):=M\left(\lambda_{i}\right) v_{i} \exp \left(\lambda_{i}\right)\right\}_{i=1, \ldots, N} .
$$

For every $M$ it is known [3] that the Pick matrix satisfies a Lyapunov matrix equation; we give a direct proof based on the calculus of QDFs.

Proposition 1: Consider $M$ partitioned as in (6), and define

$$
\begin{aligned}
\Lambda & :=\operatorname{diag}\left(\lambda_{i}\right)_{i=1, \ldots, N} \\
U & :=\left[D\left(\lambda_{i}\right) v_{i}\right]_{i=1, \ldots, N} \\
Y & :=\left[N\left(\lambda_{i}\right) v_{i}\right]_{i=1, \ldots, N} ;
\end{aligned}
$$


then $P$ satisfies the equation

$$
P \Lambda+\Lambda^{*} P=U^{*} Y+Y^{*} U .
$$

Proof:

$$
\begin{aligned}
& D(\zeta)^{\top} N(\eta)+N(\zeta)^{\top} D(\eta) \\
= & \zeta \frac{D(\zeta)^{\top} N(\eta)+N(\zeta)^{\top} D(\eta)}{\zeta+\eta} \\
+ & \eta \frac{D(\zeta)^{\top} N(\eta)+N(\zeta)^{\top} D(\eta)}{\zeta+\eta} .
\end{aligned}
$$

Substituting $\zeta$ with $\lambda_{i}{ }^{*}, \eta$ with $\lambda_{j}$, and multiplying on the left by $v_{i}^{*}$ and on the right by $v_{j}$ we obtain the claim.

In case $M$ defines a conservative port-Hamiltonian system the Pick matrix (3) enjoys the following important property.

Proposition 2: Let $X\left(\frac{d}{d t}\right)$ be a minimal state map for the system, and let $Q$ be the invertible energy matrix defined by (11). Define

$$
Z:=\left[\begin{array}{lll}
X\left(\lambda_{1}\right) v_{1} & \ldots & X\left(\lambda_{N}\right) v_{N}
\end{array}\right] \in \mathbb{C}^{n \times N} ;
$$

then

$$
P=Z^{*} Q Z \text {. }
$$

Consequently, if $N \leq n$ then $P$ is nonsingular. The maximal possible rank of $P$ is $n$.

Proof: Use Th. 1 and (11) to conclude that for all $1 \leq i, j \leq N$ it holds that $\left(M\left(\lambda_{i}\right) v_{i}\right)^{*} Q_{e} M\left(\lambda_{j}\right) v_{j}=\left(\lambda_{i}{ }^{*}+\right.$ $\left.\lambda_{j}\right)\left(X\left(\lambda_{i}\right) v_{i}\right)^{*} Q X\left(\lambda_{j}\right) v_{j}$. From this (17) follows.

We prove that $Z$ has full rank; the rest of the claims easily follows. Let $N \leq n$, and assume by contradiction that $\operatorname{rank}(Z)<N$; then there exist $\alpha_{i} \in \mathbb{C}, i=1, \ldots, N$, not all zero, such that $Z \operatorname{col}\left(\alpha_{i}\right)_{i=1, \ldots, N}=0$. Consider the trajectory $\hat{\ell}:=\sum_{i=1}^{N} \alpha_{i} v_{i} \exp \left(\lambda_{i}\right)$. Let $F \in \mathbb{R}^{m \times m}[\xi]$ be such that ker $\left(F\left(\frac{d}{d t}\right)\right)$ equals the subspace of $\mathfrak{C}^{\infty}\left(\mathbb{R}, \mathbb{R}^{m}\right)$ spanned by $v_{i} \exp \lambda_{i}, i=1, \ldots, N$; such $F$ always exists (see section $\mathrm{XV}$ of [17]). Now consider the following equations:

$$
w=M\left(\frac{d}{d t}\right) \ell, x=X\left(\frac{d}{d t}\right) \ell, 0=F\left(\frac{d}{d t}\right) \ell
$$

The external behavior $\mathfrak{B}^{\prime}$ described by these equations is autonomous (see [10]), of dimension $N$. Moreover $X\left(\frac{d}{d t}\right)$ is a state map for $\mathfrak{B}^{\prime}$, since it is a state map for $\operatorname{im} M\left(\frac{d}{d t}\right) \supset$ $\mathfrak{B}^{\prime}$. Choose $\ell=\hat{\ell}$ in (18); then the value of $\hat{x}:=X\left(\frac{d}{d t}\right) \hat{\ell}$ at $t=0$ is zero. Since $\mathfrak{B}^{\prime}$ is autonomous, it follows that $\hat{w}:=M\left(\frac{d}{d t}\right) \hat{\ell}$ is also zero. From full rank of $M$ it follows that $\hat{\ell}=0$, which is in contradiction with the assumption that not all $\alpha_{i}$ 's are equal to zero. Thus $Z$ has rank $N$.

\section{IDENTIFICATION OF CONSERVATIVE PORT-HAMILTONIAN SYSTEMS}

The result of Proposition 2 suggests that when the number of data $N$ in (13) is larger than or equal to the minimal dimension of the state space $n$, a set of state trajectories $x_{i} \exp \left(\lambda_{i}\right)$ can be computed as follows. Let $P=Z^{*} Q Z$ be a rank-revealing factorization of $P$, i.e. $Z \in \mathbb{C}^{n \times N}$ and $Q \in \mathbb{R}^{n \times n}$ have full row rank, equal to $n=\operatorname{rank} P$; then the $i$-th column of $Z$ is the required vector $x_{i} \in \mathbb{C}^{n}$.
Now assume that $N=n$; we show that the subspace of $\mathbb{C}^{2 n+2 m}$ defined by

$$
\mathcal{D}:=\operatorname{span}\left\{\left[\begin{array}{c}
u \\
Q x_{i} \\
y_{i} \\
-\lambda_{i} x_{i}
\end{array}\right]=\left[\begin{array}{c}
D\left(\lambda_{i}\right) v_{i} \\
Q X\left(\lambda_{i}\right) v_{i} \\
N\left(\lambda_{i}\right) v_{i} \\
-\lambda_{i} X\left(\lambda_{i}\right) v_{i}
\end{array}\right]\right\}_{i=1, \cdots, N}
$$

can be "completed" to a Dirac structure.

Proposition 3: Define $\mathcal{D}$ by (19). Then there exists a $m$ dimensional subspace $\mathcal{V} \subset \mathbb{C}^{2 n}$ such that $\mathcal{D} \oplus \mathcal{V}$ is a (complex) Dirac structure.

Proof: The system associated with the data $u_{i}, y_{i}$ and the corresponding state vectors $x_{i}$ is of the form (12). Consequently, $\mathcal{D}$ is contained in the graph of the skewsymmetric map defined by

$$
\begin{aligned}
L: \mathbb{C}^{n+m} & \rightarrow \mathbb{C}^{n+m} \\
{\left[\begin{array}{l}
z_{1} \\
z_{2}
\end{array}\right] } & \mapsto\left[\begin{array}{cc}
-J & -B \\
B^{\top} & M
\end{array}\right]\left[\begin{array}{l}
z_{1} \\
z_{2}
\end{array}\right]
\end{aligned}
$$

It follows (see section 1.1 of [4]) that a suitable $\mathcal{V}$ can be found so that $\mathcal{D} \oplus \mathcal{V}$ is a Dirac structure.

The following result gives a parametrization of all skewsymmetric maps whose graph contains $\mathcal{D}$. For simplicity of exposition we will assume that $Q>0$ (corresponding to a lossless port-Hamiltonian system). This means that a rankrevealing factorization of $P$ can be taken to be $P=Z^{*} Z$.

Proposition 4: Assume that $N=n$, and let $P=Z^{*} Z$ be a rank-revealing factorization of $P$. Define $U, Y$ as in (14). The following statements are equivalent:

1) The matrices $A^{\prime} \in \mathbb{R}^{n \times n}, B^{\prime} \in \mathbb{R}^{n \times m}, M^{\prime} \in \mathbb{R}^{m \times m}$ satisfy

$$
\left[\begin{array}{c}
-Z \Lambda \\
Y
\end{array}\right]=\left[\begin{array}{ll}
A^{\prime} & B^{\prime} \\
C^{\prime} & M^{\prime}
\end{array}\right]\left[\begin{array}{l}
Z \\
U
\end{array}\right]
$$

and moreover $\left[\begin{array}{cc}A^{\prime} & B^{\prime} \\ C^{\prime} & M^{\prime}\end{array}\right]$ is skew-symmetric;

2) There exist $X=-X^{\top} \in \mathbb{R}^{m \times m}$ such that

$$
\begin{aligned}
& {\left[\begin{array}{ll}
A^{\prime} & B^{\prime}
\end{array}\right]=\left[\begin{array}{ll}
-Z \Lambda Z^{-1} & 0_{N \times m}
\end{array}\right]+K\left[U Z^{-1} \quad-I_{m}\right]}
\end{aligned}
$$

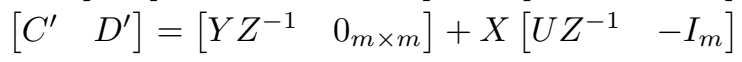

where $K:=-Z^{-*} Y^{\top}-Z^{-*} U^{\top} X^{\top}$.

Proof: It is a matter of straightforward verification to check that $A^{\prime} \in \mathbb{R}^{n \times n}, B^{\prime} \in \mathbb{R}^{n \times m}, M^{\prime} \in \mathbb{R}^{m \times m}$ satisfy (20) if and only if they are of the form (21) for some choice of arbitrary matrices $X$ and $K$. The skew-symmetry in (20) occurs if and only if $X$ is skew-symmetric, and $K=-Z^{-*} Y^{\top}-Z^{-*} U^{\top} X^{\top}$.

Remark 1: Using the terminology of ([17]), we say that the set consisting of quadruples $\left(A^{\prime}, B^{\prime}, C^{\prime}, M^{\prime}\right)$ parametrized as in statement 2) of Proposition 4 is the set of unfalsified models of the data $x_{i} \exp \left(\lambda_{i}\right),\left[\begin{array}{l}u_{i} \\ y_{i}\end{array}\right] \exp \left(\lambda_{i}\right)$, $i=1, \ldots, N$, in the model class of conservative, input-stateoutput port-Hamiltonian systems. 
Remark 2: Note that the unfalsified models are parametrized by the feedthrough term $X$, and that among the unfalsified models there exists precisely one such that $M^{\prime}=M$, the feedthrough term in (12).

Remark 3: The need for rank-revealing factorisations arises in may areas, and the symmetric case is especially relevant in signal processing and optimization theory. $\mathrm{Nu}$ merically speaking, the singular-value decomposition (SVD) is the most accurate way of performing such factorisation; however, alternative faster algorithms are available although not as informative or as accurate as the SVD. The standard reference on the SVD is the book [7]; the papers [2], [5], [8] illustrate alternative rank-revealing factorization algorithms. The application of these numerical procedures in our approach is a matter of ongoing investigation.

\section{DATA-DRIVEN REDUCED-ORDER MODELING OF CONSERVATIVE PORT-HAMILTONIAN SYSTEMS}

In the previous section we assumed that the number $N$ of trajectories of the system was equal to $n$, the minimal dimension of the state space. In this section we consider the case $N<n$, i.e. we consider a subset of $N^{\prime}<n$ data points. We partition the matrices $U$ and $Y$ and $Z$ as

$$
U=\left[\begin{array}{ll}
U_{a} & U_{b}
\end{array}\right], Y=\left[\begin{array}{ll}
Y_{a} & Y_{b}
\end{array}\right], Z=\left[\begin{array}{ll}
Z_{a} & Z_{b}
\end{array}\right]
$$

where $U_{a}, Y_{a} \in \mathbb{C}^{m \times N^{\prime}}, Z_{a} \in \mathbb{C}^{n \times N^{\prime}}$. We also partition $P$ and $\Lambda$ accordingly, i.e.

$$
P=\left[\begin{array}{ll}
P_{a a} & P_{a b} \\
P_{b a} & P_{b b}
\end{array}\right], \Lambda=\operatorname{diag}\left(\Lambda_{a}, \Lambda_{b}\right) .
$$

Straightforward verification shows that the 'reduced' Pick matrix $P_{a a} \in \mathbb{C}^{N^{\prime} \times N^{\prime}}$ satisfies the Lyapunov equation

$$
P_{a a} \Lambda_{a}+\Lambda_{a}^{*} P_{a a}=U_{a}^{*} Y_{a}+Y_{a}^{*} U_{a} .
$$

Evidently, $P_{a a}=Z_{a}^{*} Z_{a}$; now consider a rank-revealing factorization of $P_{a a}$ as

$$
P_{a a}=Z^{*} Z^{\prime}
$$

where $\operatorname{rank} P=\operatorname{rank} Z^{\prime}=: N^{\prime} \leq n$, and denote the columns of $Z^{\prime} \in \mathbb{C}^{N^{\prime} \times N^{\prime}}$ with $z_{i}, i=1, \ldots, N^{\prime}$.

We now show that the subspace of $\mathbb{C}^{2 N^{\prime}+2 m}$ defined by

$$
\mathcal{D}^{\prime}:=\operatorname{span}\left\{\left[\begin{array}{c}
u \\
z_{i}^{\prime} \\
y_{i} \\
-\lambda_{i} z_{i}^{\prime}
\end{array}\right]\right\}_{i=1, \cdots, N^{\prime}}
$$

is contained in the graph of a skew-symmetric map, and consequently can be completed to a Dirac structure. In proving this statement, we also show that among the skewsymmetric maps whose graph contains $\mathcal{D}^{\prime}$ there exists one which is obtained by projecting the matrices $J, B$, and $M$ of the data-generating representation (12).

Proposition 5: Define $\mathcal{D}^{\prime}$ by (25); there exists a skewsymmetric matrix $L \in \mathbb{R}^{\left(N^{\prime}+m\right) \times\left(N^{\prime}+m\right)}$ such that $\mathcal{D}^{\prime}$ is contained in the graph of $L$. Moreover, one such $L$ is

$$
L=\left[\begin{array}{cc}
-Z^{\prime-*} Z_{a}^{*} J Z_{a} Z^{\prime-1} & -Z^{\prime-*} Z_{a}^{*} B \\
-B^{\top} Z_{a} Z^{\prime-1} & M
\end{array}\right]
$$

where $Z_{a}$ is defined by (22), $Z^{\prime}$ is any matrix arising from a rank-revealing factorisation (24) of $P_{a a}$, and $J, B$ and $M$ are as in (12).

Proof: Since $P_{a a}=Z_{a}^{*} Z_{a}=Z^{*} Z^{\prime}$, and since $Z^{\prime}$ is nonsingular, we write

$$
Z^{\prime}=Z^{\prime-*} Z_{a}^{*} Z_{a}
$$

From this equation it follows that

$$
\begin{aligned}
-Z^{\prime} \Lambda & =-Z^{\prime-*} Z_{a}^{*} Z_{a} \Lambda=Z^{\prime-*} Z_{a}^{*} \cdot\left(-J Z_{a}-B U\right) \\
& =-Z^{\prime-*} Z_{a}^{*} J Z_{a}-Z^{\prime-*} Z_{a}^{*} B U \\
& =-\underbrace{Z^{\prime-*} Z_{a}^{*} J Z_{a} Z^{\prime-1}}_{=: J^{\prime}} Z^{\prime}-\underbrace{Z^{\prime-*} Z_{a}^{*} B}_{=: B^{\prime}} U .
\end{aligned}
$$

Observe that $J^{\prime}$ is skew-symmetric.

Since $Y=C Z_{a}+M U$ where $C=-B^{\top}$, it follows that

$$
Y=-\underbrace{B^{\top} Z_{a} Z^{\prime-1}}_{=B^{\prime \top}} Z^{\prime}+D U=B^{\prime \top} Z^{\prime}+M U .
$$

Consequently, $\mathcal{D}^{\prime}$ is contained in the graph of the skewsymmetric map defined by (26).

It follows from the considerations gathered in Remark 1 and Remark 2 that the reduced-order representation associated with the skew-symmetric matrix $L$ defined in (26) is precisely that associated with the choice of $X=-M$, where $X$ is the parameter defined in Prop. 4, and $M$ is the feedthrough term of the data-producing system equations (12). The following result is a straightforward consequence of these considerations.

Corollary 1: Define $\mathcal{D}^{\prime}$ by (25). A skew-symmetric matrix $L \in \mathbb{R}^{\left(N^{\prime}+m\right) \times\left(N^{\prime}+m\right)}$ whose graph contains $\mathcal{D}^{\prime}$ is $L=$ : $\left[\begin{array}{cc}L_{11} & L_{12} \\ -L_{12}^{\top} & L_{22}\end{array}\right]$, where

$$
\begin{aligned}
L_{11} & :=-Z^{\prime} \Lambda Z^{\prime-1}-Z^{\prime-\top} Y^{\top}-Z^{\prime-\top} U^{\top} M U Z^{\prime-1} \\
L_{12} & :=-Z^{\prime-\top} Y^{\top}-Z^{\prime-\top} U^{\top} M \\
L_{22} & :=M .
\end{aligned}
$$

\section{ThE SELF-ADJOINT HAMILTONIAN CASE}

In this section we will sketch how the theory developed in the previous sections can be extended to self-adjoint Hamiltonian systems. This entails the introduction of a skewsymmetric version of the Pick matrix, and the replacement of Dirac structures by Lagrangian subspaces, see Appendix VIII.

\section{A. Self-adjoint Hamiltonian systems}

Consider on the space of outputs and inputs the standard symplectic form defined by

$$
\Omega_{e}=\left[\begin{array}{cc}
0_{m} & I_{m} \\
-I_{m} & 0_{m}
\end{array}\right]
$$

Then a system given in image representation (5) is called a self-adjoint Hamiltonian system if

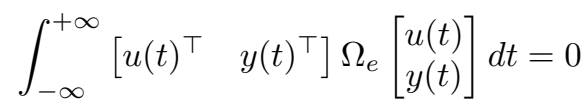


for all compact-support trajectories $\operatorname{col}(u, y)$. It can be shown [16] that this holds if and only if there exists $\Psi \in$ $\mathbb{R}^{m \times m}[\zeta, \eta]$ such that

$$
\begin{aligned}
M(\zeta)^{T} \Omega_{e} M(\eta) & =D(\zeta)^{T} N(\eta)-N(\zeta)^{T} D(\eta) \\
& =(\zeta+\eta) \Psi(\zeta, \eta)
\end{aligned}
$$

We obtain the following statement paralleling Theorem 1.

Theorem 2: Let $X\left(\frac{d}{d t}\right)$ be a minimal state map for the system represented by (5). Assume that the system is selfadjoint Hamiltonian, and let $Q_{\Psi}$ be its storage function. There exists an invertible $\Omega=-\Omega^{\top} \in \mathbb{R}^{n \times n}$ such that for all $(\operatorname{col}(u, y), \ell)$ satisfying (5) and corresponding $x:=X\left(\frac{d}{d t}\right) \ell$, it holds that $Q_{\Psi}(w)=\left(X\left(\frac{d}{d t}\right) \ell\right)^{\top} \Omega\left(X\left(\frac{d}{d t}\right) \ell\right)$.

It can be shown [16] that the state space representation of a self-adjoint Hamiltonian system is given as

$$
\begin{aligned}
\Omega \frac{d}{d t} x & =-Q x-C^{T} u \\
y & =C^{\top} x+M u
\end{aligned}
$$

where $J=-J^{\top} \in \mathbb{R}^{n \times n}, Q=Q^{\top} \in \mathbb{R}^{n \times n}$, and $M=M^{\top} \in \mathbb{R}^{m \times m}$. Here $\frac{1}{2} x^{T} Q x$ represents the internal Hamiltonian of the system. It follows that

$$
\frac{d}{d t} x_{1}^{\top} \Omega x_{2}=u_{1}^{\top} y_{2}-y_{1}^{\top} u_{2}
$$

showing that instead of an energy balance as in (11) the fundamental property of any self-adjoint Hamiltonian system is the relation between the internal symplectic form $\Omega$ with the external symplectic form $\Omega_{e}$. Note in this respect that, since $\Omega$ has full rank, there always exist state space coordinates (called canonical coordinates) in which $\Omega$ takes the form

$$
\Omega=\left[\begin{array}{cc}
0_{n} & I_{n} \\
-I_{n} & 0_{n}
\end{array}\right]
$$

\section{B. The skew-symmetric Pick matrix}

The above system class motivates to introduce the following skew-symmetric version of the Pick matrix. Consider as before $N$ pairs $\left(\lambda_{i}, v_{i}\right), i=1, \ldots, N$, where $v_{i} \in \mathbb{C}^{m}$, $\lambda_{i} \in \mathbb{C}$ with the property that $\lambda_{i}$ does not lie on the imaginary axis, $\lambda_{i} \neq \lambda_{j}$ for $i \neq j$, and $\lambda_{j}=\lambda_{i}^{*} \Rightarrow v_{j}=\overline{v_{i}}$, with data-set defined by (13).

Define instead of the standard Pick matrix the skewsymmetric Pick matrix as the skew-Hermitian matrix whose $(i, j)$-th entry is

$$
S_{i, j}:=\frac{\left[\begin{array}{ll}
u_{i}^{*} & y_{i}^{*}
\end{array}\right]\left[\begin{array}{cc}
0_{m \times m} & I_{m} \\
-I_{m} & 0_{m \times m}
\end{array}\right]\left[\begin{array}{l}
u_{i} \\
y_{i}
\end{array}\right]}{\lambda_{i}^{*}+\lambda_{j}} .
$$

Also the skew-symmetric Pick matrix $S$ defined from this data set satisfies a Lyapunov matrix equation. In fact for any (not necessarily self-adjoint Hamiltonian) system we have

Proposition 6: Consider $M$ partitioned as in (6), and define $\Lambda, U, Y$ as in (14) Then the skew-symmetric Pick matrix $S$ defined by (34) satisfies

$$
S \Lambda+\Lambda^{*} S=U^{*} Y-Y^{*} U .
$$

Proof:

$$
\begin{aligned}
& D(\zeta)^{\top} N(\eta)-N(\zeta)^{\top} D(\eta) \\
= & \zeta \frac{D(\zeta)^{\top} N(\eta)-N(\zeta)^{\top} D(\eta)}{\zeta+\eta} \\
+ & \eta \frac{D(\zeta)^{\top} N(\eta)-N(\zeta)^{\top} D(\eta)}{\zeta+\eta} .
\end{aligned}
$$

Substituting $\zeta$ with $\lambda_{i}{ }^{*}, \eta$ with $\lambda_{j}$, and multiplying on the left by $v_{i}^{*}$ and on the right by $v_{j}$ we obtain the claim.

For a self-adjoint Hamiltonian system the skew-symmetric Pick matrix (34) enjoys the following property paralleling Proposition 2.

Proposition 7: Let $X\left(\frac{d}{d t}\right)$ be a minimal state map for the system, and let $\Omega$ be the symplectic matrix defined by (32). Define

$$
Z:=\left[\begin{array}{lll}
X\left(\lambda_{1}\right) v_{1} & \cdots & X\left(\lambda_{N}\right) v_{N}
\end{array}\right] \in \mathbb{C}^{n \times N} ;
$$

then

$$
P=Z^{*} \Omega Z
$$

Consequently, if $N \leq n$ then $P$ is nonsingular. The maximal possible rank of $P$ is $n$.

\section{Identification of self-adjoint Hamiltonian systems}

The identification theory for self-adjoint Hamiltonian systems parallels the one for conservative port-Hamiltonian systems. Let $S=Z^{*} \Omega Z$ be a rank-revealing factorization of $S$, i.e. $Z \in \mathbb{C}^{n \times N}$ and $\Lambda \in \mathbb{C}^{n \times n}$ have full row rank, equal to $n=\operatorname{rank} S$; then the $i$-th column of $Z$ is the required vector $x_{i} \in \mathbb{C}^{n}$. It follows that (35) can be written in the following form

$$
Z^{*}(\Omega Z) \Lambda-\Lambda^{*}(\Omega Z)^{*} Z=U^{*} Y-Y^{*} U
$$

This means that the Dirac structure formulation as given before for conservative port-Hamiltonian systems is replaced by a Lagrangian subspace formulation. (See the Appendix VIII for the definition of a complex Lagrangian subspace.)

Indeed, assume as before that $N=n$; then we show that the subspace of $\mathbb{C}^{2 n+2 m}$ defined by

$$
\mathcal{L}:=\operatorname{span}\left\{\left[\begin{array}{c}
u_{i} \\
x_{i} \\
y_{i} \\
-\lambda_{i} \Omega x_{i}
\end{array}\right]=\left[\begin{array}{c}
D\left(\lambda_{i}\right) v_{i} \\
X\left(\lambda_{i}\right) v_{i} \\
N\left(\lambda_{i}\right) v_{i} \\
-\lambda_{i} \Omega X\left(\lambda_{i}\right) v_{i}
\end{array}\right]\right\}_{i=1, \cdots, N}
$$

can be "completed" to a Lagrangian subspace.

Proposition 8: Define $\mathcal{L}$ by (39). Then there exists a $m$ dimensional subspace $\mathcal{V} \subset \mathbb{C}^{2 n}$ such that $\mathcal{L} \oplus \mathcal{V}$ is a Lagrangian subspace.

Proof: The system associated with the data $u_{i}, y_{i}$ and the corresponding state vectors $x_{i}$ is of the form (31). Consequently, $\mathcal{L}$ is contained in the graph of the symmetric map defined by

$$
\left[\begin{array}{l}
z_{1} \\
z_{2}
\end{array}\right] \mapsto\left[\begin{array}{cc}
Q & B \\
B^{\top} & M
\end{array}\right]\left[\begin{array}{l}
z_{1} \\
z_{2}
\end{array}\right]
$$


It follows that a suitable $\mathcal{V}$ can be found so that $\mathcal{L} \oplus \mathcal{V}$ is a Lagrangian subspace.

A parametrisation of all symmetric maps whose graph contains $\mathcal{L}$ can be given along the same lines as the parametrisation given for the conservative port-Hamiltonian case in Proposition 4. Also the data-driven reduced-order modelling of self-adjoint Hamiltonian systems can be performed in a similar way. Because of space limitations, we will not enter into such details.

\section{CONCLUSiOnS}

Our approach to system identification and model order reduction is based on exploiting the connection between the external structure of the system, associated with a bilinear form on the inputs and outputs, and its internal structure, associated with a bilinear form on the state variables. If the external structure is known, state trajectories corresponding to the given data can be computed factorising certain constant matrices computed from the data.

Several issues are a matter of ongoing investigation. We need to clarify the relation of the approach presented here to other ones based on interpolation (see [1]), or specifically used for port-Hamiltonian systems (see [11]). We need to study the approximating properties (for example error-bounds) of the reduced-order systems defined by our procedures. In order for our method to be practical in real application, we need to investigate the numerically robust and computationally efficient implementation of the algorithms (see Remark 3). Finally, we need to investigate the potential of this approach to deal with dissipative systems.

\section{APPENDIX}

For completeness we slightly adapt the definition of a Dirac structure as given in [4], [15], [14] to a real linear space $\mathcal{V}$ with inner product $\langle\cdot \mid \cdot\rangle$. Define on $\mathcal{V} \times \mathcal{V}$ the bilinear form

$$
\ll\left(f^{a}, e^{a}\right),\left(f^{b}, e^{b}\right) \gg:=\left\langle e^{a} \mid f^{b}\right\rangle+\left\langle e^{b} \mid f^{a}\right\rangle,
$$

with $\left(f^{a}, e^{a}\right),\left(f^{b}, e^{b}\right) \in \mathcal{V} \times \mathcal{V}$. Then $\mathcal{D} \subset \mathcal{V} \times \mathcal{V}$ is a Dirac structure with respect to the bilinear form $\ll \cdot, \cdot \gg$ if $\mathcal{D}=\mathcal{D}^{\Perp}$, where ${ }^{\Perp}$ is with respect to $\ll \cdot, \cdot \gg$. For a finitedimensional linear space $\mathcal{V}$ this is equivalent to $\operatorname{dim} \mathcal{D}=$ $\operatorname{dim} \mathcal{V}$ and $\langle e \mid f\rangle=0$ for all $(f, e) \in \mathcal{D}$.

This definition can be extended to the complex case as follows.

Definition 2: Consider $\mathbb{C}^{n}$ (or any linear space over $\mathbb{C}$ ) with complex inner product $\langle\cdot \mid \cdot\rangle$. Define on $\mathbb{C}^{n} \times \mathbb{C}^{n}$ the sesquilinear form

$$
\ll\left(f^{a}, e^{a}\right),\left(f^{b}, e^{b}\right) \gg:=\left\langle e^{a} \mid f^{b}\right\rangle+\left\langle e^{b} \mid f^{a}\right\rangle,
$$

with $\left(f^{a}, e^{a}\right),\left(f^{b}, e^{b}\right) \in \mathbb{C}^{n} \times \mathbb{C}^{n}$, and $\langle\cdot \mid \cdot\rangle$ the complex inner product. Then $\mathcal{D} \subset \mathbb{C}^{n} \times \mathbb{C}^{n}$ is a complex Dirac structure if $\mathcal{D}=\mathcal{D}^{\Perp}$ where $\Perp$ is with respect to the sesquilinear form. This is equivalent to $\operatorname{dim} \mathcal{D}=n$ and $\langle e \mid f\rangle=0$ for all $(f, e) \in \mathcal{D}$.
Similarly, a complex Lagrangian subspace is defined as follows:

Definition 3: Consider $\mathbb{C}^{n}$ (or any linear space over $\mathbb{C}$ ) with complex inner product $\langle\cdot \mid \cdot\rangle$. Define on $\mathbb{C}^{n} \times \mathbb{C}^{n}$ the sesquilinear form

$$
\ll\left(f^{a}, e^{a}\right),\left(f^{b}, e^{b}\right) \gg:=\left\langle e^{a} \mid f^{b}\right\rangle-\left\langle e^{b} \mid f^{a}\right\rangle,
$$

with $\left(f^{a}, e^{a}\right),\left(f^{b}, e^{b}\right) \in \mathbb{C}^{n} \times \mathbb{C}^{n}$. Then $\mathcal{L} \subset \mathbb{C}^{n} \times \mathbb{C}^{n}$ is a complex Lagrangian subspace if $\mathcal{L}=\mathcal{L}^{\Perp}$, where ${ }^{\Perp}$ is with respect to the sesquilinear form. This implies $\operatorname{dim} \mathcal{L}=n$.

\section{REFERENCES}

[1] Astolfi, A., "Model Reduction by Moment Matching for Linear and Nonlinear Systems", IEEE Trans. Aut. Contr., vol. 55, no.10, pp. 23212336, 2010.

[2] BAKER, E.S. AND R.D. DEGROAT, "A correlation-based subspace tracking algorithm", IEEE Trans. Sign. Proc., vol.46, no.11, pp. 31123116, 1998.

[3] J.A. BALL, I. Gohberg and L. RodmAn, Interpolation of Rational Matrix Functions, Birkhäuser Verlag, Basel Boston Berlin, 1990.

[4] Courant, T.J. "Dirac manifolds", Trans. AMS, vol. 319, no. 2, pp. 631-661, 1990.

[5] Dopico, F. M. AND P. KoEv, "Accurate symmetric rank revealing and eigendecompositions of symmetric structured matrices", SIAM J. Matr. Anal. Appl., vol. 28, no. 4, pp. 1126-1156, 2006.

[6] The Geoplex Consortium, Modeling and Control of Complex Physical Systems: the Port-Hamiltonian Approach. Springer-Verlag, Berlin Heidelberg, 2009.

[7] Golub, G. And C.F. VAn LoAn, Matrix Computations, North Oxford Academic, 1983.

[8] Hansen, P.C. and P. Y. Yalamov, "Computing Symmetric RankRevealing Decompositions Via Triangular Factorization", SIAM J. Matr. Anal. Appl., vol. 23, no. 2, pp. 443-458, 2001.

[9] VAN OverscheE, P. and DE MOOR, B., Subspace identification for linear systems, Kluwer, Dordrecht, 1996.

[10] J.W. POLDERMAN and J.C. WILlEMS, Introduction to mathematical system theory: a behavioral approach, Springer-Verlag, Berlin, 1997.

[11] POLYuga, R.V. and A.J. VAN DER SCHAFT, "Structure preserving model reduction of port-Hamiltonian systems by moment matching at infinity", Automatica, vol. 46, no. 4, pp. 665-672, 2010.

[12] Rapisarda, P. and H.L. Trentelman, "Identification and datadriven model reduction of state-space representations of lossless and dissipative systems from noise-free data", Automatica, vol. 47, pp. 1721-1728, 2011.

[13] P. RAPISARDA and J.C. WILlEMS, "State maps for linear systems", SIAM J. Control Opt., vol. 35, nr. 3, pp. 1053-1091, 1997.

[14] A.J. VAN DER SCHAFT, $L_{2}$-Gain and Passivity Techniques in Nonlinear Control, Springer Communications and Control Engineering series. Springer-Verlag, London, 2nd revised and enlarged edition, 2000.

[15] A.J. VAN DER SchafT and B. MAschKe, "The Hamiltonian formulation of energy conserving physical systems with external ports", Archiv für Elektronik und Übertragungstechnik, vol. 49, pp. 362-371, 1995.

[16] A.J. VAN DER SCHAFT and P. RAPISARDA, "State maps from integration by parts", SIAM J. Control Opt., vol. 49, no. 6, pp. 2415-2439, 2011.

[17] Willems, J.C., "Paradigms and puzzles in the theory of dynamical systems", IEEE Trans. Aut. Contr., vol. 36, pp. 259-294, 1991.

[18] Willems, J.C. and H.L. Trentelman, "On quadratic differential forms", SIAM J. Control Opt., vol. 36, pp. 1703-1749, 1998. 